Allergie-Kongress 2004, Aachen Hyposensibilisierung - neue
Option bei Neurodermitis?

\author{
Der Einsatz der Hyposensibilisierung bei Patienten mit allergischen \\ Atemwegserkrankungen, die zusätzlich unter einem atopischen \\ Ekzem leiden, wird kontrovers diskutiert. Viele Ärzte befürchten eine \\ Verschlimmerung des Ekzems. Zwei deutsche Studien haben diese \\ Einstellung jetzt auf den Prüfstand gestellt.
}

B ei Patienten mit atopischem Ekzem setzen manche Allergologen die spezifische Immuntherapie (SIT) auch dann nur sehr zurückhaltend ein, wenn die Behandlung wegen einer gleichzeitigen allergischen Atemwegserkrankung sinnvoll wäre. Hintergrund: Immer wieder wird gemutmaßt, dass eine SIT die Neurodermitis verschlechtert.

\section{Ekzem-Exazerbation befürchtet}

Für diese Vermutung gibt es durchaus auch pathophysiologisch sinnvolle Begründungen, erklärte Priv.-Doz. Dr. Ulf Darsow, München: So führt die SIT zu einem passageren Anstieg der IgE-Spiegel, welcher das Ekzem verschlimmern könnte. Weiter ähneln sich die Zytokinmuster beim Umschalten von der TH2zur TH1-Immunantwort bei Hyposensibilisierung und Neurodermitis, eine Chronifizierung des Ekzems wird befürchtet. Beide Annahmen sind plausibel,

Voraussetzungen für eine Hyposensibilisierung des atopischen Ekzems

1. Aeroallergene kommen als signifikante Triggerfaktoren des atopischen Ekzems in Betracht.

2. Die individuelle Relevanz des Allergens sollte durch geeignete diagnostische Maßnahmen nachgewiesen sein.

3. Eine Hyposensibilisierungsmethode mit erwiesener Effizienz sollte verfügbar sein, die eine signifikante Verbesserung im Krankheitsverlauf des atopischen Ekzems induziert. der Zusammenhang aber ist letztlich nicht bewiesen.

Die bisherigen Studien zur SIT bei Neurodermitis kranken vor allem an der kleinen Fallzahl, viele sind eher kasuistische Darstellungen. In der Zusammenschau, so Darsow, hätte die Hyposensibilisierung aber bei immerhin sechs der insgesamt neun vorliegenden plazebokontrollierten Untersuchungen das Ekzem positiv beeinflusst. Die neuesten Daten haben im vergangenen Jahr zwei deutsche Studien geliefert.

\section{SIT schadet nicht ...}

Dr. Darsow berichtete über eine multizentrische Phase-III-Studie, an der er selbst beteiligt war: Insgesamt 44 Patienten mit atopischem Ekzem und einer gleichzeitigen Sensibilisierung gegen Hausstaubmilben erhielten randomisiert und doppelblind ein Jahr lang entweder eine orale Immuntherapie mit einem Milbenextrakt oder Plazebotropfen. Als ein Ansprechen auf die Therapie waren eine Reduktion des SCORAD um wenigstens $20 \%$ und eine Reduktion der symptomatischen Medikation um wenigstens 40\% definiert. Diese Kriterien erfüllten am Ende 17 von 20 Patienten in der SITGruppe und 15 von 17 in der Plazebogruppe. Insgesamt lag die Wirksamkeit der oralen Immuntherapie also auf Plazeboniveau, andererseits führte sie aber auch nicht zu einer Exazerbation des Ekzems.

\section{... sondern nützt vielleicht sogar}

Deutlich erfolgreicher war die Hyposensibisierung beim atopischen Ekzem in einer aktuellen Studie einer Arbeitsgruppe um Prof. Dr. Alexander Kapp, Han-

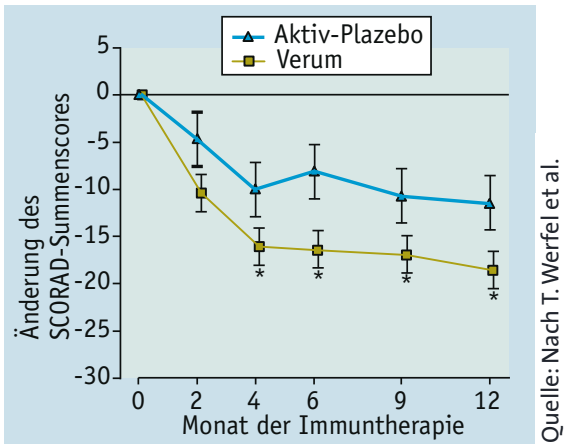

Veränderung des SCORAD bei Patienten mit atopischem Ekzem und einer Sensibilisierung gegen Hausstaubmilben nach einer 12-monatigen Hyposensibilisierung - * $p<0,05$

nover: 79 Patienten mit mittelschwerer bis schwerer Neurodermitis, die ebenfalls gegen Hausstaubmilben sensibilisiert waren, wurden in drei Gruppen eingeteilt. Alle Gruppen erhielten eine spezifische subkutane Immuntherapie mit einem Milbenextrakt, die erste mit einer Erhaltungsdosis von 20, die zweite mit 2.000 und die dritte mit 20.000 SQ-Einheiten. Die erste Gruppe mit der geringen Allergenkonzentration wurde als „Aktiv-Plazebogruppe“ betrachtet und mit den beiden Hochdosisgruppen verglichen. Am Ende des einjährigen Studienzeitraums war der SCORAD in den beiden Hochdosisgruppen signifikant stärker zurückgegangen als in der Vergleichsgruppe (Abb.). Außerdem mussten die Patienten in den beiden SIT-Gruppen signifikant weniger topische Glukokortikoide anwenden.

\section{Versuchsweiser Einsatz möglich}

Insgesamt, so Darsows Fazit, müssten die Bedenken, dass eine SIT die Symptome des atopischen Ekzems verstärkt, nun teilweise revidiert werden. Ob die Hyposensibilisierung darüber hinaus tatsächlich auch eine neue Option zur Behandlung der Neurodermitis ist, bleibt fraglich. Die aktuelle Evidenz reiche noch nicht aus, um eine klare Empfehlung abzugeben. Bei individueller Indikationsstellung kann aber ein probeweiser Einsatz schon jetzt versucht werden. es

Darsow UG. SIT beim atopischen Ekzem. Allergie-Kongress 2004, Aachen, 15.-19.

September 2004 